University of Nebraska - Lincoln

DigitalCommons@University of Nebraska - Lincoln

\title{
Arena Size Modulates Functional Responses via Behavioral Mechanisms
}

Stella F. Uiterwaal

University of Nebraska - Lincoln, suiterwaal@huskers.unl.edu

Anthony I. Dell

National Great Rivers Research and Education Center

John P. DeLong

University of Nebraska - Lincoln, jpdelong@unl.edu

Follow this and additional works at: https://digitalcommons.unl.edu/bioscifacpub

Part of the Biology Commons, and the Ecology and Evolutionary Biology Commons

Uiterwaal, Stella F.; Dell, Anthony I.; and DeLong, John P., "Arena Size Modulates Functional Responses via Behavioral Mechanisms" (2019). Faculty Publications in the Biological Sciences. 828.

https://digitalcommons.unl.edu/bioscifacpub/828

This Article is brought to you for free and open access by the Papers in the Biological Sciences at DigitalCommons@University of Nebraska - Lincoln. It has been accepted for inclusion in Faculty Publications in the Biological Sciences by an authorized administrator of DigitalCommons@University of Nebraska - Lincoln. 
Uiterwaal, Dell, and DeLong in Behavioral Ecology (2019) 30(2): 483-489. doi:10.1093/beheco/ary188.

Copyright 2019, OUP. Used by permission.

\title{
Arena Size Modulates Functional Responses via Behavioral Mechanisms
}

\author{
Stella F. Uiterwaal, Anthony I. Dell, and John P. DeLong \\ SFU, JPD: School of Biological Sciences, University of Nebraska-Lincoln, Lincoln, Nebraska, USA \\ AID: National Great Rivers Research and Education Center (NGRREC), East Alton, Illinois, USA \\ Address correspondence to SFU, suiterwaal@huskers.unl.edu
}

\begin{abstract}
Laboratory-based functional response experiments, in which foraging rates are measured across a range of resource densities, are central for determining trophic interaction strength. Historically these experiments often are performed in arbitrarily sized arenas, with larger sized organisms generally used in larger arenas. However, arena size influences foraging rates and therefore also estimates of the functional response parameters, particularly space clearance rate (attack rate). We hypothesized that nonrandom movement within arenas by predators and prey may explain this effect. To test this hypothesis, we video-recorded Schizocosa ocreata wolf spiders (predators) and flightless Drosophila melanogaster prey in circular arenas of 3 different sizes to reveal thigmotactic behavior. We then estimated foraging rates and space clearance rates from feeding trials performed at a single, low prey density in 3 differently-size arenas in either annular (ring-shaped) or circular arenas. Annular arenas mitigated the effects of predator and prey aggregation and thus controlled the experienced prey density near arena edges. Unlike the circular arenas, annular arenas produced similar foraging rates and space clearance rate estimates across arena sizes, confirming that it is the increased density of prey along edges that generates the previously observed arena size effect. Our results provide a key insight into how animal behavior and experimental design must be considered for the accurate interpretation of foraging rates, both when considering standalone functional responses and when making comparisons across experiments.
\end{abstract}

Keywords: Arena size, Density, Drosophila melanogaster, Functional response, Schizocosa ocreata, Space clearance rate, Thigmotaxis, Tracking

\section{Introduction}

Understanding consumer-resource interactions is essential to many basic and applied questions in ecology (Novak and Wootton 2010; Rall et al. 2012; Alexander et al. 2014; Uiterwaal and DeLong 2018). There is a long history of quantifying predator-prey interactions using functional response experiments, which relate the foraging rate of consumers to prey density. Since their development almost 60 years ago (Holling 1959), functional responses have grown to now represent a central tool for ecologists, with results from these studies having fundamentally influenced diverse issues in ecology, from trophic interaction strength (Vucic-Pestic et al. 2010) to invasive species management (Dick et al. 2017; Laverty et al. 2017), and even the evaluation of biocontrol agents (Kalinkat and Rall 2015; Uiterwaal and DeLong 2018). Therefore, a fuller understanding of the factors that influence functional responses has broad implications for ecology ( $\mathrm{Li}$ et al. 2018).

A key difference between functional response experiments and the field conditions to which experimental results are often extrapolated is that in functional response experiments predator and prey are bounded within an experimental arena, which is generally not the case in nature. Understanding the ways that experimental arena design, including size and shape, influence our estimation of foraging rates is therefore critical to obtaining accurate estimates of field foraging rates, which are essential for a fuller and more accurate understanding of ecological systems. Recent work has revealed that the outcome of functional responses depends on arena size, with space clearance rate (also known as attack rate) increasing with the total size of the arena (Yaşar and Özger 2005; Uiterwaal et al. 2017). In fact, arena size may be even more important in driving space clearance rate than other well-established factors, such as environmental temperature and body size (Uiterwaal and DeLong 2018). Given that functional responses are often performed in somewhat arbitrarily sized arenas, failure to quantitatively account for arena size means that measures of trophic interaction strength are confounded by laboratory techniques. Furthermore, this means that arena size must be accounted for in order to compare functional response parameters across experiments. To do this, we must understand the mechanisms underlying the arena size effect. Simulations suggest that foraging rates change as arena size changes, but it is not clear how this change affects space clearance rate (Li et al. 2017).

Received August 1, 2018; revised November 13, 2018; editorial decision November 19, 2018; accepted November 30 , 2018; advance access publication December 21, 2018; uploaded to UNL institutional repository January 2021. 
A common form of the functional response-known as Type II-is the Holling disc equation:

$$
f=\frac{a R}{1+a h R}
$$

where $f$ is the per capita foraging rate of the predator (in units of $\frac{\text { prey }}{\text { pred time }}$ ), $a$ is the space clearance rate (how quickly a predator removes prey from a given area) $\left(\frac{\text { space }}{\text { pred } * \text { time }}\right), h$ is handling time (the time cost for each prey consumed) ( $\left.\frac{\text { time }}{\text { prey }}\right)$, and $R$ is the initial resource density ( $\left.\frac{\text { prey }}{\text { space }}\right)$ (Holling 1959). The use of resource space densities to predict foraging rate using a functional response assumes that the random movement of individuals minimizes any spatial heterogeneity in their distribution, which if present could alter predator-prey encounters and thus the observed functional response (Fryxell et al. 2007). In other words, in a randomly distributed system, consumer-resource encounters should occur with equal probability at all locations throughout the arena (Holling 1959). In fact, it is not just the Type II Holling disc equation (Equation 1) that makes this assumption. Many functional response forms, including other types (e.g. I or III), and the random predator equation (Royama 1971; Rogers 1972), are built around this tenet of random motion.

However, evidence from the literature suggests that predators and prey may not distribute themselves randomly in experimental arenas, and that the way predators and prey use space is important in determining functional responses (Kaiser 1983; McKenzie et al. 2012). For example, many organisms show a tendency to seek physical contact with objects such as arena edges, a process known as positive thigmotaxis (Fraenkel and Gunn 1961). In this sense, thigmotaxis serves as a mechanism for prey animals to find shelter and avoid predation (Antonelli et al. 1999), which may explain why arena complexity changes functional responses (Hoddle 2003; Hauzy et al. 2010; Toscano and Griffen 2013). Predators also can exploit thigmotactic behavior to their advantage: African wild dogs use fence lines to herd prey and facilitate hunting (Dyk and Slotow 2003). If thigmotaxis occurs in species used in functional response experiments, then the violation of the random distribution assumption in Equation 1 could explain the surprising effect of arena size on space clearance rate (Uiterwaal et al. 2017; Uiterwaal and DeLong 2018).

In short, if positive thigmotaxis exists, the underutilization of space in the center of arenas could result in a discrepancy between calculated prey density (initial number of prey provided divided by total arena area) and experienced prey density (density experienced by the predator) because both predator and prey are concentrated along the edges of arenas. This thigmotaxis effect is shown in Figure 1. At the calculated (true) prey density (A), a predator's actual foraging rate (B) is given by its functional response (solid black line). The space clearance rate for this curve is shown as a tangent to the functional response as it approaches the origin (solid grey line). However, thigmotactic behavior of prey and predators would result in a higher experienced density $(C)$, which produces a higher foraging rate (D). This higher foraging rate is then

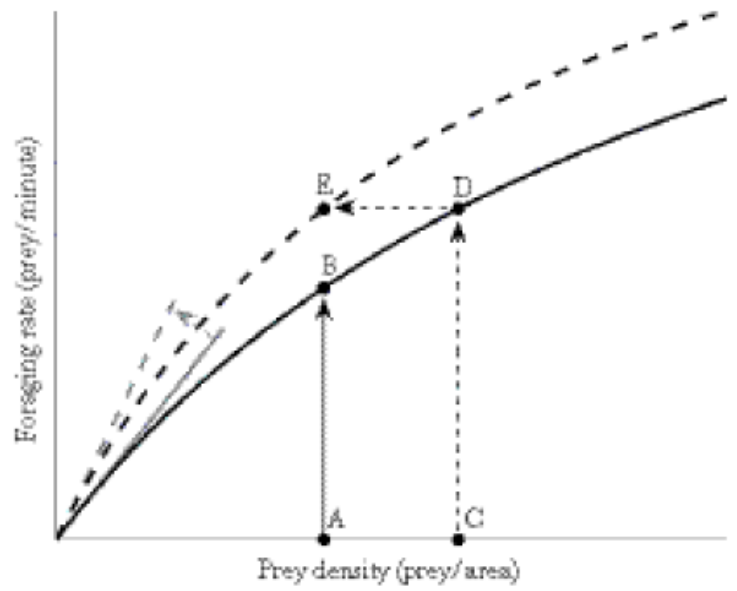

Figure 1

Thigmotaxis would result in increased estimates of space clearance rate (grey lines), which determines the initial rise of the functional response (black lines). At the calculated density (A), a predator's actual foraging rate (B) is given by its functional response (solid black line) and space clearance rate is relatively low (solid grey line). However, thigmotactic behavior of prey and predators would result in a higher experienced density $(\mathrm{C})$ around the arena edges. This would result in more encounters between predators and prey, producing a higher foraging rate (D). This is erroneously plotted (E) against a lower density (A), leading to an increased estimate of space clearance rate (dashed grey line) and altering the calculated functional response (dashed black line). Note that, at higher prey densities, the effect of thigmotactic behavior on foraging rates would become vanishingly small as handling time, not space clearance, rate becomes the primary constraint. This may explain why handling time is minimally affected by arena size (cite). This figure also demonstrates how foraging rates at very low prey densities can be used to estimate space clearance rate. Because the initial rise of the functional response is determined by space clearance rate, we can use foraging rate over prey density to calculate the initial slope as a proxy for space clearance rate.

Table 1

Arena sizes and shapes used in foraging trials

$\begin{array}{llcc}\text { Arena size } & \text { Arena shape } & \text { Outer diameter }(\mathrm{cm}) & \text { Area }\left(\mathrm{cm}^{2}\right) \\ \text { Small } & \text { Circular } & 9.0 & 63.62 \\ & \text { Annular } & 9.0 & 54.00 \\ \text { Medium } & \text { Circular } & 14.5 & 165.13 \\ & \text { Annular } & 14.5 & 101.51 \\ \text { Large } & \text { Circular } & 25.0 & 490.87 \\ & \text { Annular } & 25.0 & 207.35\end{array}$

erroneously paired (E) with the calculated density (A), leading to an increased estimate of space clearance rate (dashed grey line) and altering the calculated functional response (dashed black line). Thus, the key assumption of the random distribution of resources in Equation 1 may be violated when predator-prey interactions are contained within arenas.

The discrepancy between calculated and experienced prey densities should increase with arena size because the area of a circle increases faster than the perimeter as radius 
increases (Table 1). Thus, more prey aggregate along the perimeter in larger arenas, increasing the thigmotaxis effect and resulting in the observed positive link between arena size and space clearance rate. Here, we describe results of experiments designed to explicitly test these ideas. First, we used automated tracking (Dell et al. 2014) of predators (the wolf spider Schizocosa ocreata) and prey (the fruit fly Drosophila melanogaster) to determine if individuals distribute themselves randomly throughout circular experimental arenas of different sizes (Hypothesis 1). We then tested how, if present, thigmotaxis influenced estimates of the functional response by performing foraging trials at a single, low prey density in annular (i.e., ring-shaped) arenas and calculating foraging rates and estimating space clearance rates in these arenas. Annular arenas prevented individuals from accessing the central area of the arena and thus reduced the total amount of unused space in our resource density calculations. We predicted that similar numbers of prey would be consumed in circular and annular arenas with the same outer diameter containing the same number of prey, despite their very different actual prey densities (Hypothesis 2). We further predicted that the spiders' space clearance rates in annular arenas would remain constant regardless of arena size, due to the dominant effect of the total length of arena edges and not total area (Hypothesis 3). Lastly, we predicted that space clearance rates in circular and annular arenas would be most similar at small diameters, where the amount of underutilized space in the center of circular arenas - and therefore the difference between calculated and experienced density-is minimized (Hypothesis 4). As the outer diameter of the arena increases, so should the difference between space clearance rates in circular and annular arenas.

\section{Methods}

We used the wolf spider S. ocreata as our model predator, collecting them at night from grasslands at the University of Nebraska's Cedar Point Biological Station (Ogallala, Nebraska). Spiders used in our trials had a mean mass of $37 \mathrm{mg}$ (range: $21-57 \mathrm{mg}$ ). Wingless D. melanogaster obtained from a commercial supplier served as prey. We conducted all experiments in the evening in June 2017 under dim lighting and at temperatures of approximately $22^{\circ} \mathrm{C}$. We used round plastic arenas of 3 sizes: small ( $9 \mathrm{~cm}$ outer diameter), medium (14.5 $\mathrm{cm})$, and large $(25 \mathrm{~cm})$ (Table 1$)$. We treated arena walls with Fluon, which made the walls too slick for spiders and flies to climb on. This prevented escape and constrained movement to 2 dimensions.

\section{Video tracking of movement}

To test Hypothesis 1, we conducted trials in 3 sizes of circular arenas to determine how arena size affected the movement and distribution of organisms. Predator trials contained a single spider per arena, regardless of arena size. Prey trials used either 6, 17, or 49 flies in small, medium, or large arenas, respectively, to provide a standardized density of $\sim 0.01$ flies per $\mathrm{cm}^{2}$. We used 6 replicate trials for all 6 treatments, except the trial comprising 6 prey in the small arena, which only had 5 replicates, for a total of 35 trials. We placed arenas on $45.7 \times 45.7 \mathrm{~cm}$ backlit surfaces that emitted even and diffuse IR light at $850 \mathrm{~nm}$ wavelength (Smartvision Lights, Muskegon, Michigan, USA). A single infrared-sensitive video camera (Basler acA 1300 - 60gmNIR, Basler AG, Ahrensburg, Germany) positioned $\sim 75 \mathrm{~cm}$ above each arena facing downwards onto the experimental arena recorded movements. We recorded videos using StreamPix7 software (NorPix, Toronto, Canada) at 15 frames per second for $30 \mathrm{~min}$ (to match the trial time of foraging experiments) and analyzed each video using the automated tracking program Ctrax (Branson et al. 2009), which provided an estimate of the location of the mid-point of each animal in each frame. From these tracking data, we then used Matlab to determine in every frame the shortest distance between each individual's location and the arena edge and created frequency distributions of distance to edge for each arena size.

\section{Foraging trials}

We conducted foraging trials in both circular and annular arenas. Annular arenas were identical to circular arenas, except for a plastic circle placed in the center of the arena within which organisms could not enter. The inner circle's radius was always $2 \mathrm{~cm}$ smaller than the arena's outer radius, and so organisms were confined to a $2 \mathrm{~cm}$ wide ring along the outer edges of each arena, irrespective of arena size.

In small, medium, and large circular arenas, we added 6,17 , and 49 flies, respectively, in addition to a single spider. This yielded $\sim 0.01$ flies per $\mathrm{cm}^{2}$ in all circular arenas, regardless of size. We performed 2 types of trials in the annular arenas. In the first set of trials, we added a spider and the same number of prey as in circular arenas: 6, 17, and 49 flies in small, medium, and large arenas, respectively. This created a situation equivalent to the circular arenas, except that both predator and prey were restricted to a $2 \mathrm{~cm}$ wide band between the outer and inner circles (in essence, forcing thigmotactic behavior) (Hypothesis 2). In the second set of foraging trials in annular arenas, we used the same prey density as in the circular arenas, 0.01 flies per $\mathrm{cm}^{2}$ (Hypotheses 3 and 4). To these arenas, we added 5, 10, and 21 flies for small, medium, and large annular arenas respectively. These trials simulated a situation where prey were distributed randomly in space as predicted by traditional assumptions of functional responses; this arena shape allowed for the random distribution of animals despite thigmotactic behavior because they could never be far from an edge. We performed a total of 54 trials comprising 3 arena sizes, 3 trial types $(1$ in circular arenas and 2 in annular arenas), and 6 replicates per treatment.

Note that, from Equation $1, \frac{f}{R}=\frac{a}{1+a h R^{\prime}}$ as prey density $(R)$ approaches zero, $\frac{f}{R}$ approaches space clearance rate, illustrating how space clearance rate determines the initial slope of the functional response. Thus, by using a low prey density, we were able to use foraging rate divided by prey density $\left(\frac{f}{R}\right)$ as a proxy for space clearance rate. This simplification is valid with 2 caveats. Firstly, prey density must be sufficiently small. The prey density used here $\left(0.01\right.$ flies per $\left.\mathrm{cm}^{2}\right)$ is sufficiently low to lie along the initial steep slope (Figure 1) of a typical Lycosidae functional responses (Hardman and Turnbull 1974; Monzó et al. 2009; Vucic-Pestic et al. 2010; Rall et al. 2011). Secondly, space clearance rate estimates obtained using this proxy can only be compared if the foraging rates are measured at the same density. In the first set of trials in annular arenas, prey density was not consistent between circular and annular arenas. Thus, we were only able to compare foraging rates (Hypothesis 2), not space clearance rate differences. However, the second set of trials in annular arenas (where starting density was always 0.01 flies per $\mathrm{cm} 2$ ) allowed us to test for an effect of arena size on space clearance rate in annular arenas (Hypothesis 3). Additionally, because the calculated prey density in these annular 
arenas was the same as in the circular arenas, these trials allowed us to compare space clearance rates in circular and annular arenas (Hypothesis 4). Although we could have simply used foraging rate as a proxy for space clearance rate in these cases, we wanted to highlight the differences between the first set of trials in annular arenas (where prey number is the same in circular arenas and only foraging rates can be compared) and the second set of trials in annular arenas (where prey density is the same as in circular arenas and we can compare space clearance rates).

Before the foraging trials commenced, we fed spiders to satiation then starved them for $48 \mathrm{~h}$ to standardize hunger levels. We initiated trials by placing flies haphazardly throughout the arena where they acclimated and moved freely for $10 \mathrm{~min}$. Then, we placed a single spider in each arena and allowed it to forage undisturbed for $30 \mathrm{~min}$. We chose this (relatively short) trial time for 2 reasons. Firstly, we wanted to minimize chances of complete prey depletion, which could potentially result in underestimates of space clearance rate. Alternatively, we could have replaced prey as they were consumed during the experiment, but this would have disturbed the animals and interrupted their natural movement patterns. Secondly, we chose a 30-min trial time because wolf spiders catch many prey items in quick succession and gather them into a "meatball" which is masticated and externally digested (Kiritani et al. 1972; Nyffeler and Benz 1988). Our observations indicated that once spiders had developed a satisfactorily large meatball, their foraging behavior changed and they were less likely to attack prey. A short trial time decreased the chances of spiders collecting sufficient prey to become "satiated." From the initial video trials to characterize movement and distribution of organisms (see above), we observed that fly mortality due to causes other than predation was absent, and so the number of live flies remaining at the end of each trial was a good indicator of predator consumption rate. We performed analysis of covariances (ANCOVAs) on both feeding rate and space clearance rate using arena size and shape as predictor variables.

\section{Results}

Our first set of trials to determine how spiders and flies distribute themselves in circular arenas revealed a strong positive thigmotactic response, with both species spending a disproportionate amount of time closer to the arena edge than the center. The magnitude of this effect was largely independent of arena size (Figures 2 and 3) (Hypothesis 1). Both spiders and flies spent most of their time within $2 \mathrm{~cm}$ of the arena edges (Figure 3).

As predicted, when the same number of prey was given in annular and circular arenas of the same size, spider foraging rates did not differ between arena shapes $(t=0.85, P=$ 0.400) (Figure 4a, Hypothesis 2), even though total available area (and thus calculated densities) in annular arenas was much less than in equivalently sized circular arenas (Table 1). In contrast, the effect of arena size on space clearance rates depended on arena shape (size $\times$ shape interaction: $t=2.54, P$ $=0.016)$. Estimated space clearance rates increased with arena size for circular arenas $(t=4.70, P<0.001)$, but not for annular arenas $(t=1.11, P=0.276)$.

\section{Discussion}

Results from our initial distribution experiments clearly revealed that both spiders and flies did not distribute themselves randomly across circular arenas, thus violating a basic assumption of functional response models (Holling 1959). Individuals of both species exhibited a strong positive thigmotactic response and spent the vast majority of their time close to the arena edges (Figures 2 and 3) (Hypothesis 1). Flies tended to be closer to the edge of the arena than spiders (Figure 2), with the proportion of time spent at a given location for both species decreasing farther from the edge. This behavior did not vary with arena size, suggesting that distance from the edge, and not distance to center, was the factor guiding space use (i.e., positive thigmotaxis).

We speculate that differences between spiders and flies in how close they tend to be to arena edges is determined by some combination of body size and detection distance, with larger species (i.e., spiders) able to maintain a further distance from the edge and still detect it. This size-dependent detection distance may result from either visual or physical sensing of the arena edge. Larger organisms often have better visual acuity (McGill and Mittelbach 2006), allowing spiders to be farther from the edge than the flies and still see the edge. In terms of physical detection, spiders (which have a larger body size

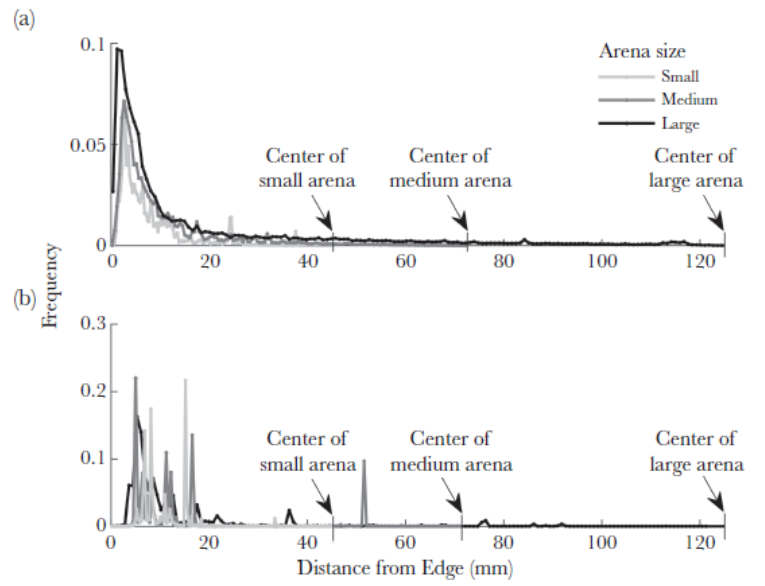

Figure 2

Frequency distributions of the locations of prey (Drosophila melanogaster) (a) and predators (Schizocosa ocreata) (b) in small, medium, and large circular arenas. 

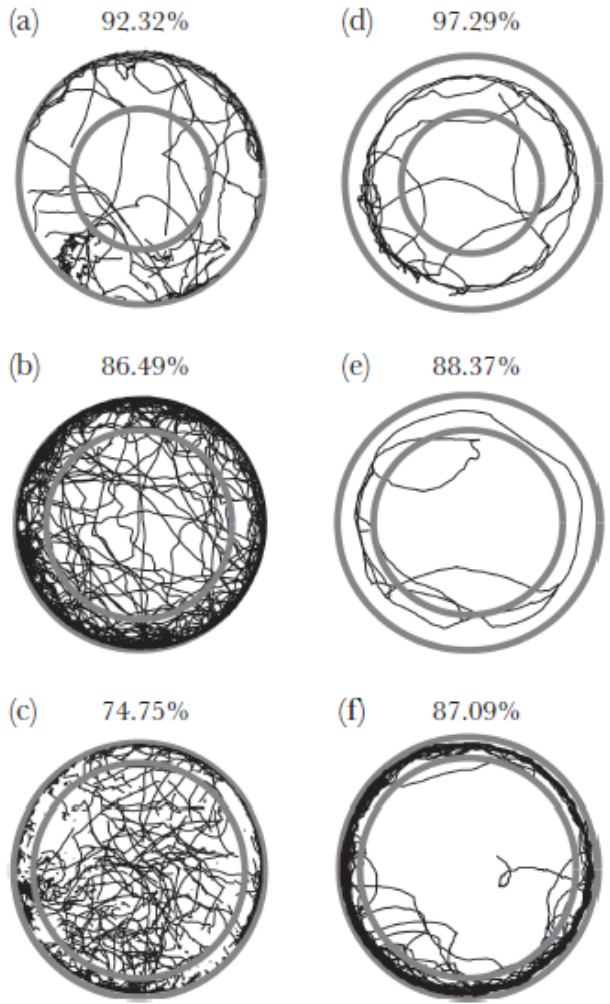

Figure 3

Sample tracks of Drosophila melanogaster prey (a, b, and c) and an Schizocosa ocreata predator arena $(\mathrm{d}, \mathrm{e}$, and $\mathrm{f})$ in small (a and $\mathrm{d})$, medium (b and e), and large (c and f) circular arenas. Outer grey circles represent arena edges. Inner grey circles are drawn $2 \mathrm{~cm}$ from arena edges. Percentages indicate proportion of time spent within $2 \mathrm{~cm}$ of the arena edges for all 6 replicates in each treatment.

and therefore longer limbs) may have seemed farther away from the arena edge than flies even if both had limbs in physical contact with the edge, since Ctrax records to the position of an organism's center.

The decrease in frequency of occupancy beyond 2 centimeters from the wall was more abrupt for spiders than for the flies, and within the 2 centimeters, the frequency distributions were not as smooth for spiders as they were for flies. This is likely due to testing 6 spiders at each arena size, while dozens (in the smaller arenas) and hundreds (in the larger arenas) of flies were tested. The central area, which normally would be considered in density calculations, remained largely empty of both predators and prey, although it became progressively more utilized in smaller arenas.

We further predicted that the number of prey eaten in circular and annular arenas of the same outer diameter would be the same if the same number of prey were initially present, despite their very different total areas available (Table 1) (Hypothesis 2). Our results supported this hypothesis (Figure 4a). Visual observation of the foraging trials confirmed our results from the video tracking experiment: spiders and flies remained near arena edges even when they had access to the center. Thus, preventing access to the center with the internal rings had minimal effect on the distribution and movement of predators or prey, and therefore foraging rates, despite large differences in the total area available. Because both spiders and prey preferred the edges even when they had access to the center, experienced prey density in the circular arenas was effectively similar to calculated prey density in annual arenas. Although calculated densities were very different in circular and annular arenas, foraging rates were largely identical because experienced densities were largely identical. This suggests that, for functional response experiments conducted in larger arenas, observed foraging rates may be matched with erroneously low calculated densities, inflating space clearance rate estimates (Figure 1).

As predicted, space clearance rate increased with arena size in the circular arenas. In annular arenas, however, space clearance rate was not significantly different across arena sizes (Figure 4b) (Hypothesis 3). This clearly shows that accounting for underused space with annular arenas can mitigate the effect of arena size on space clearance rates.

Lastly, our results confirmed our prediction that the difference between space clearance rates in circular and annular arenas would be lowest in the smallest arenas and increase as arena size increases (Hypothesis 4). Because space clearance rate increased with arena size in circular arenas but was largely unaffected by arena size in annular arenas, the space clearance rate disparity increased with arena size (Figure $4 b$ ). This suggests that space clearance rates obtained from functional responses conducted in small arenas are most accurate. However, at some very small diameter the amount of space outside of the predator's immediate detection distance presumably is minimized to the point where searching is no longer necessary and predators no longer display normal foraging behavior.

Our results show that thigmotactic behavior in foraging arenas results in an increased experienced prey density even when calculated density remains constant. Although the amount of occupied space also increases with arena size, the proportion of unused to used space increases with arena size because a circle's area grows faster than its perimeter. Thus, by preferring the edges, both prey and predators approach a 1-dimensional orientation in which arena circumference is more important than arena area. That is, the increased experienced density is a result of reduced dimensionality. Consequently, although we typically calculate density as prey per area, it may be more appropriate to use prey per length (i.e., circumference in circular arenas). If both predators and prey move predominantly along the outer edges of the arena, this would give a more accurate representation of prey abundance. However, because the spiders and flies tested here used a $2 \mathrm{~cm}$ band along the edge of the arena, animal movement was not entirely restricted to one dimension. The actual prey density was somewhere between the calculated linear density (number of prey divided by circumference) and the calculated area density (number of prey divided by total arena area).

To account for the effects of thigmotaxis, we suggest that obtaining consistent estimates of space clearance rate requires accounting for arena size in 1 of 4 ways: 1) Using annular arenas to eliminate differences between experienced and calculated prey density, 2) Ignoring any unused space in circular arenas when calculating density, 3) Using small arenas to minimize unused space, or 4) Correcting the space clearance rate estimates to a common standard arena size using statistical relationships between space clearance rate and arena size (Uiterwaal and DeLong 2018). 
UiterWAAL, Dell, AND DELONG IN BEHAVIORAL ECOLOGY (2019) 30(2): 483-489.

(a)

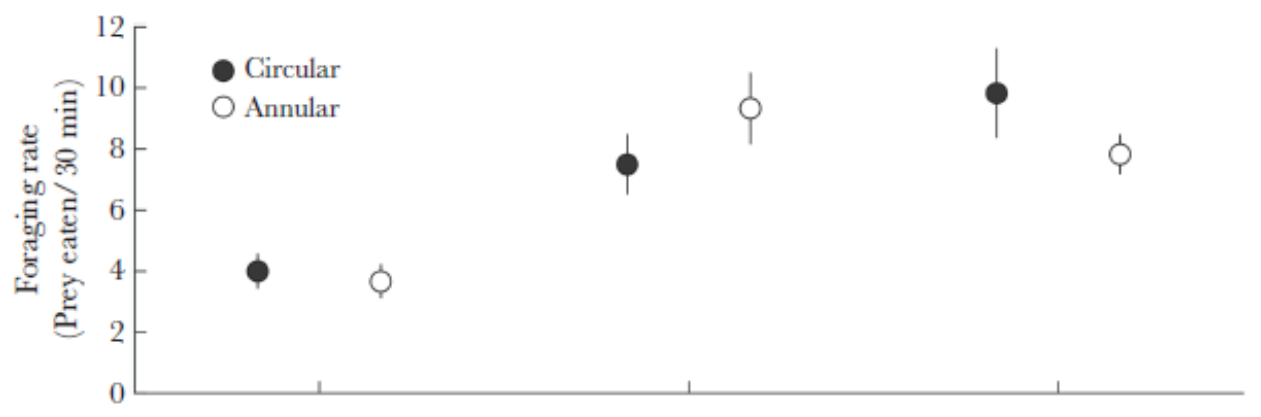

(b)

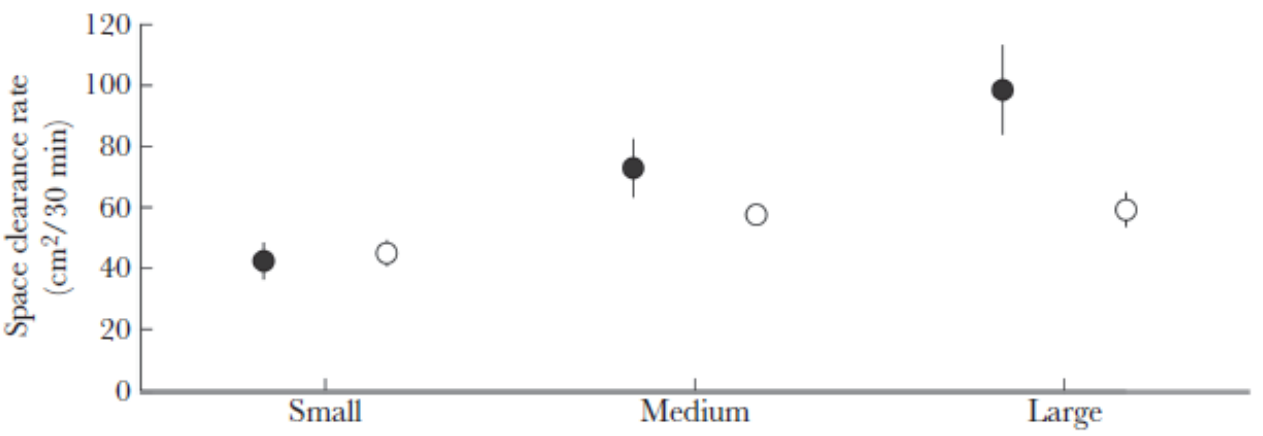

Figure 4

Mean $( \pm S E)$ foraging rate (a, predators exposed to same prey number, Hypothesis 2$)$ and space clearance rate (b, predators exposed to same prey density, Hypotheses 3 and 4) of Schizocosa ocreata foraging on Drosophila melanogaster in circular and annular arenas of various sizes.

Alternatively, using linear density (i.e., prey per edge length) to describe prey abundances may be appropriate in arenas with radii much wider than the outer band of space used by predators and prey.

Perhaps more important is the need to consider thigmotaxis in predator-prey interactions outside of laboratory conditions. In the field, foraging space is not limited to empty arenas and impassable walls; real foraging habitats are considerably more complex. Accordingly, many studies have looked at the effects of habitat complexity (Hohberg and Traunspurger 2005; Hauzy et al. 2010; Kalinkat et al. 2013; Toscano and Griffen 2013; Barrios-O'Neill et al. 2016) and edge structure (Kaiser 1983; Hoddle 2003) on functional responses. Such studies typically find that increased complexity and structure reduces predator feeding rates. Although we were unable to add habitat complexity to our trials without compromising tracking ability, we predict that placing sheltering structures throughout the arena (perhaps in such a way that animals can never be more than $2 \mathrm{~cm}$ from shelter) may encourage random distribution and eliminate any effect of arena size. However, Vucic-Pestic et al. (2010) found the opposite: prey distributed randomly in empty arenas but aggregated when structure was introduced.

Clearly, more work is required to elucidate how thigmotactic behavior interacts with habitat structure in arenas. Nonetheless, we suggest that the addition of structure may favor natural behaviors of both predators and prey, producing functional responses that can more accurately be extrapolated to the field. This is true regardless of whether structure promotes random distribution or induces aggregation. We further suggest that the same predator may have radically different foraging rates depending on the habitat structure of its immediate surroundings. Thus, spatial heterogeneity in natural habitats may be more important in structuring food webs than previously thought. Indeed, physical edges may play a role in determining interaction strengths in food web links wherever habitat structure occurs.

Funding: This material is based on work supported by a National Science Foundation Graduate Research Fellowship (grant no. DGE1610400) to SFU.

Acknowledgments: We thank Jon Garbisch and Cedar Point Biological Station for facilitating this work, and Ashley Olson and Carl Cloyed for assisting with the experiment.

Authors' contributions: S.F.U. conducted the experiments and analyzed the data. SFU and JPD wrote the initial draft. All authors contributed to final editing of the manuscript.

Data accessibility: Analyses reported in this article can be reproduced using the data provided by Uiterwaal et al. (2018).

Handling editor: John Skelhorn.

\section{References}

Alexander ME, Dick JT, Weyl OL, Robinson TB, Richardson DM. 2014. Existing and emerging high impact invasive species are characterized by higher functional responses than natives. Biol Lett. 10:20130946.

Antonelli J, Steele C, Skinner C. 1999. Cover-seeking behavior and shelter use by juvenile and adult Crayfish, Procambarus clarkii: potential importance in species invasion. J Crustac Biol. 19:293-300. Barrios-O'Neill, D, Kelly, R, Dick, JTA, Ricciardi, A, MacIsaac, HJ, Emmerson, MC. 2016. On the context-dependent scaling of consumer feeding rates. Ecol Lett. 19:668-678. 
Branson K, Robie AA, Bender J, Perona P, Dickinson MH. 2009. Highthroughput ethomics in large groups of Drosophila. Nat Methods. 6:451-457.

Dell AI, Bender JA, Branson K, Couzin ID, de Polavieja GG, Noldus LP, Pérez-Escudero A, Perona P, Straw AD, Wikelski M, et al. 2014. Automated image-based tracking and its application in ecology. Trends Ecol Evol. 29:417-428.

Dick, JTA, Laverty, C, Lennon, JJ, Barrios-O'Neill, D, Mensink, PJ, Britton, JR, Médoc, V, Boets, P, Alexander, ME, Taylor, NG, et al. 2017. Invader relative impact potential: a new metric to understand and predict the ecological impacts of existing, emerging and future invasive alien species. J Appl Ecol. 54:1,259-1,267.

Dyk G van, Slotow R. 2003. The effects of fences and lions on the ecology of African wild dogs reintroduced to Pilanesberg National Park, South Africa. Afr. Zool. 38:79-94.

Fraenkel GS, Gunn DL. 1961. The Orientation of Animals. Dover.

Fryxell JM, Mosser A, Sinclair AR, Packer C. 2007. Group formation stabilizes predator-prey dynamics. Nature 449:1041-1043.

Hardman JM, Turnbull AL. 1974. The interaction of spatial heterogeneity, predator competition and the functional response to prey density in a laboratory system of Wolf Spiders (Araneae: Lycosidae) and Fruit Flies (Diptera: Drosophilidae). J Anim Ecol. 43:155-171.

Hauzy C, Tully T, Spataro T, Paul G, Arditi R. 2010. Spatial heterogeneity and functional response: an experiment in microcosms with varying obstacle densities. Oecologia 163:625-636.

Hoddle MS. 2003. The effect of prey species and environmental complexity on the functional response of Franklinothrips orizabensis: a test of the fractal foraging model. Ecol. Entomol. 28:309-318.

Hohberg K, Traunspurger W. 2005. Predator-prey interaction in soil food web: functional response, size-dependent foraging efficiency, and the influence of soil texture. Biol Fertil Soils 41:419-427.

Holling CS. 1959. Some characteristics of simple types of predation and parasitism. Can. Entomol. 91:385-398.

Kaiser H. 1983. Small scale spatial heterogeneity influences predation success in an unexpected way: model experiments on the functional response of predatory mites (Acarina). Oecologia 56:249-256.

Kalinkat G, Brose U, Rall BC. 2013. Habitat structure alters top-down control in litter communities. Oecologia 172:877-887.

Kalinkat G, Rall B. 2015. Effects of climate change on the interactions between insect pests and their natural enemies. In Climate Change and Insect Pests, pp. 74-91. CABI. doi:10.1079/9781780643786.0074.

Kiritani K, Kawahara S, Sasaba T, Nakasuji F. 1972. Quantitative evaluation of predation by spiders on the green rice leafhopper, Nephotettix cincticeps Uhler, by a sight-count method. Res Popul Ecol. 13:187-200.

Laverty C, Green KD, Dick JTA, Barrios-O'Neill D, Mensink PJ, Médoc V, Spataro T, Caffrey JM, Lucy FE, Boets P, et al. 2017. Assessing the ecological impacts of invasive species based on their functional responses and abundances. Biol Invasions 19:1653-1665.

Li Y, Brose U, Meyer K, Rall BC. 2017. How patch size and refuge availability change interaction strength and population dynamics: a combined individual- and population-based modeling experiment. Peer] 5:e2993.

Li Y, Rall BC, Kalinkat G. 2018. Experimental duration and predator satiation levels systematically affect functional response parameters. Oikos 127:590-598.

McGill BJ, Mittelbach GG. 2006. An allometric vision and motion model to predict prey encounter rates. Evol Ecol Res. 8:691-701.

McKenzie HW, Merrill EH, Spiteri RJ, Lewis MA. 2012. How linear features alter predator movement and the functional response. Interface Focus. 2:205-216.

Monzó C, Mollá Ó, Castañera P, Urbaneja A. 2009. Activity-density of Pardosa cribata in Spanish citrus orchards and its predatory capacity on Ceratitis capitata and Myzus persicae. BioControl 54:393-402.

Novak M, Wootton JT. 2010. Using experimental indices to quantify the strength of species interactions. Oikos 119:1057-1063.

Nyffeler M, Benz G. 1988. Feeding ecology and predatory importance of wolf spiders (Pardosa spp.) (Araneae, Lycosidae) in winter wheat fields. J Appl Entomol. 106:123-134.

Rall BC, Brose U, Hartvig M, Kalinkat G, Schwarzmüller F, VucicPestic O, Petchey OL. 2012. Universal temperature and body-mass scaling of feeding rates. Philos Trans R Soc Lond B: Biol Sci. 367:2923-2934.

Rall BC, Kalinkat G, Ott D, Vucic-Pestic O, Brose U. 2011. Taxonomic versus allometric constraints on non-linear interaction strengths. Oikos 120:483-492.

Rogers D. 1972. Random search and insect population models. J Anim Ecol. 41:369-383.

Royama T. 1971. A comparative study of models for predation and parasitism. Res Popul Ecol. 13:1-91.

Toscano BJ, Griffen BD. 2013. Predator size interacts with habitat structure to determine the allometric scaling of the functional response. Oikos 122:454-462.

Uiterwaal SF, Dell A, DeLong JP. 2018. Data from: arena size modulates functional responses via behavioral mechanisms. Dryad Digital Repository. http://dx.doi.org/10.5061/dryad.4sr5b16

Uiterwaal SF, DeLong JP. 2018. Multiple factors, including arena size, shape the functional responses of ladybird beetles. J Appl Ecol. $55: 2,429-2,438$

Uiterwaal SF, Mares C, DeLong JP. 2017. Body size, body size ratio, and prey type influence the functional response of damselfly nymphs. Oecologia 185:339-346.

Vucic-Pestic O, Birkhofer K, Rall BC, Scheu S, Brose U. 2010. Habitat structure and prey aggregation determine the functional response in a soil predator-prey interaction. Pedobiologia 53:307-312.

Vucic-Pestic O, Rall BC, Kalinkat G, Brose U. 2010. Allometric functional response model: body masses constrain interaction strengths. J Anim Ecol. 79:249-256.

Yaşar B, Özger S. 2005. Functional response of Oenopia conglobata (L.) (Coleoptera: Coccinellidae) on Hyalopterus pruni (Geoffroy) (Homoptera: Aphididae) in three different size arenas. Turk. J. Entomol. 29:91-99. 